\title{
Numerical simulation of the oscillations of non-Newtonian viscous fluids with a free surface
}

A. KOROBEINIKOV

Department of Mathematics, University of Auckland, Private Bag 92019, Auckland, New Zealand

\begin{abstract}
Non-Newtonian fluids are increasingly being transported by a variety of vehicles. It has been observed that vehicles containing such fluids demonstrate behaviour which could be explained only by non-linearity characteristics of such fluids. In this paper small oscillations of non-Newtonian fluids in tanks are considered. A numerical method suitable for both Newtonian and non-Newtonian fluids is suggested. Examples of numerical simulations with emphasis on Bingham fluids are given to demonstrate effects of non-linear properties.
\end{abstract}

Keywords: non-Newtonian fluids, oscillations, free surface, numerical simulations.

\section{Introduction}

Non-Newtonian fluids are those for which shear stress do not depend on shear rate linearly (as it does for Newtonian fluid), i.e. the viscosity of a non-Newtonian fluid is not constant. Perhaps the simplest non-Newtonian fluids are Bingham plastic fluids, which differ from Newtonian fluids only in that their linear relationship between shear stress and shear rate does not go through the origin (see Fig. 1). A flow curve of a Bingham plastic fluid is a straight line with the yield stress $\tau_{0}$ which must be exceeded to initiate flow. Such materials as water suspensions of rock grains, slurries, drilling muds, oil paints, toothpaste and sewage sludge exhibit Bingham-plastic behaviour [8].

Other types of time-independent non-Newtonian fluids are pseudoplastic and dilatant fluids (see Fig. 1). Pseudoplastics perhaps include the majority of nonNewtonian fluids and are characterised by apparent viscosity decreasing with increasing shear rate. In general the flow curve of pseudoplastics are straight lines on a logarithmic plot. They include polymeric solutions and melts, suspensions of paper pulp or pigments. Dilatant materials exhibit rheological behaviour opposite to that of pseudoplastics - their apparent viscosity increases with increasing shear rate. Some examples of dilatant materials are starch or mica suspensions in water, quicksand and beach sand.

Non-Newtonian fluids are frequently encountered in industry and there is an increasing need for their transportation by specially designed vehicles. Practice shows that the non-linear viscosity of liquid load can affect motion of a vehicle itself. For instance, practically all construction mixtures such as liquid cement, concrete etc. clearly exhibit non-linear rheological characteristics [5]. It is usual practice to prepare construction mixtures at specialised plants and transport them 


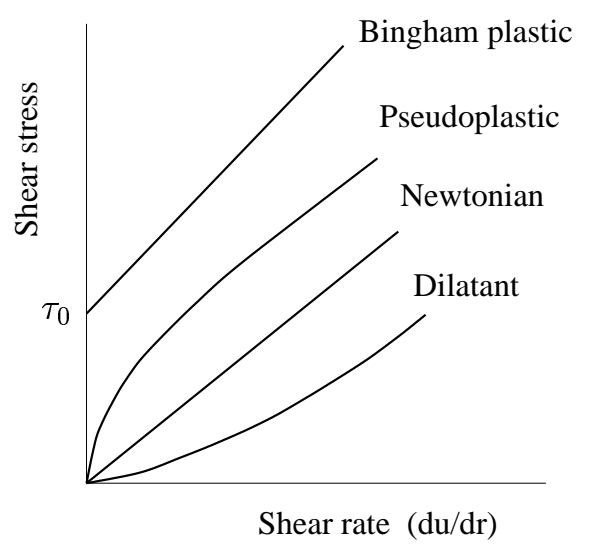

Figure 1. Flow curves for main types of time-independent non-Newtonian fluids.

to construction sites by motor vehicles. Trucks with a load of liquid concrete sometimes exhibit behaviour which can be explained only by the non-linear viscosity of the transported liquid. If we take into account that the weight of the commercial load is comparable with that of the vehicle itself and that this cargo is highly movable, while the speed of the vehicles as well as their load tends to increase, then the importance of the problem becomes obvious.

By now, due to its tremendous importance for rockets and space crafts, the dynamics of solid bodies containing fluids is an extensively developed field (e.g. see [3], [4] and many others). The theory of bodies containing ideal (inviscid) liquid is well developed area. Considerable progress have been made for Newtonian fluids as well. This leads us to the question whether this theory can be applied to the case of bodies containing non-Newtonian fluids and where are the limitations for such an application.

The aim of this paper is to explore the qualitative differences of the behaviour of Newtonian and non-Newtonian fluids in a tank and to investigate effects of nonlinear rheological properties of fluids on their dynamics. We consider only the comparatively simple problem of small oscillations of a non-Newtonian fluid with a free surface. Since our aim is mainly to demonstrate the qualitative difference of the behaviour of Newtonian and non-Newtonian fluids and to explore the effects provided by the non-linear viscosity, we will consider here two-dimensional flows only.

The numerical method used in this paper is not limited by the choice of rheological equation although in fact we will consider mainly Bingham plastic fluid. This is because the Bingham fluids exhibit behaviour which is distinctly different from that of Newtonian fluids and can serve as an indicator for comparison. The concept of an idealised Bingham plastic is also very convenient because many real fluids, such as concrete mixtures, closely approximate this type of behaviour. 


\section{Formulation of the problem}

Assume that an incompressible viscous fluid fills an open horizontal channel. We will consider oscillations of the fluid in a cross-section of the channel. A rectangular coordinate system $O x y$ is connected with the channel so that the axis $O x$ is horizontal and coincides with an undisturbed free surface $\Sigma_{0}$ and the axis $O y$ is upward and normal to $\Sigma_{0}$ (see Fig. 2).

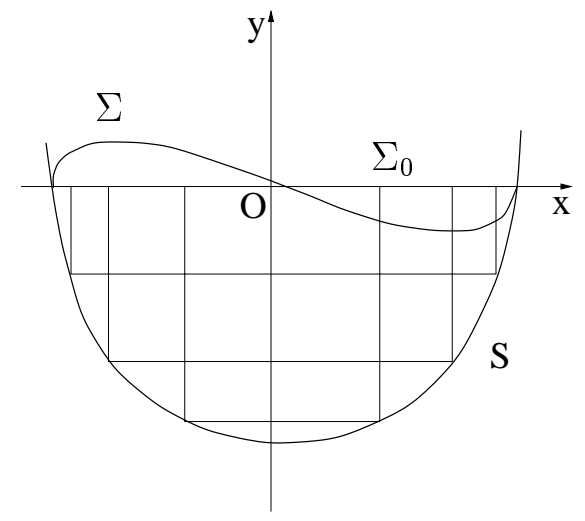

Figure 2. Channel cross-section and finite-difference mesh.

Let us assume that amplitudes of oscillations are small and motion is slow enough to justify the neglect of the convective inertia terms, which are of second order, in the equations of fluid motion (see [3], [4] for details). Then conservation of momentum is expressed as

$$
\begin{aligned}
& \rho \frac{\partial u}{\partial t}=-\frac{\partial p}{\partial x}+\frac{\partial \tau_{x x}}{\partial x}+\frac{\partial \tau_{x y}}{\partial y} \\
& \rho \frac{\partial v}{\partial t}=-\frac{\partial p}{\partial y}+\frac{\partial \tau_{x y}}{\partial x}+\frac{\partial \tau_{y y}}{\partial y}-\rho g
\end{aligned}
$$

where $u, v$ are velocity components, $\rho$ is fluid density, $p$ is pressure, $g$ is the acceleration due to gravity, and $\tau_{x x}, \tau_{x y}, \tau_{y y}$ are stresses due to fluid viscosity. The equations (1), (2) must be solved simultaneously with the equation of continuity for incompressible flow

$$
\frac{\partial u}{\partial x}+\frac{\partial v}{\partial y}=0
$$

and with the rheological (constitutive) equation which relates the viscous stress tensor $\tau$ to the fluid deformation rate tensor $\dot{\gamma}$.

E.P. Shulman [6], [7] suggested a very convenient for applications (and especially for multidimensional flows) constitutive relationship which generalises several sim- 
pler models.According to Shulman

$$
\tau=2 B \dot{\gamma}
$$

where

$$
B=\left(\frac{\tau_{0}}{A^{1 / m}}+\mu^{1 / m}\right)^{n} A^{n / m-1}
$$

is the apparent viscosity,

$$
A=\left(2 \dot{\gamma}_{x x}^{2}+4 \dot{\gamma}_{x y}^{2}+2 \dot{\gamma}_{y y}^{2}\right)^{\frac{1}{2}}
$$

is the intensity (the second invariant) of the deformation rate tensor and $\tau_{0}, \mu, m, n$ are positive parameters. The components of the deformation rate tensor $\dot{\gamma}$ are given by

$$
\dot{\gamma}_{x x}=\frac{\partial u}{\partial x}, \quad \dot{\gamma}_{y y}=\frac{\partial v}{\partial y}, \quad \dot{\gamma}_{x y}=\frac{1}{2}\left(\frac{\partial u}{\partial y}+\frac{\partial v}{\partial x}\right) .
$$

This constitutive relationship comprises a variety of rheological models which can be obtained by a prescribed choice of the parameters $\tau_{0}, \mu, m, n$. For instance, assuming that $m=n=1$ we get the Bingham rheological equation

$$
\tau=2\left(\frac{\tau_{0}}{A}+\mu\right) \dot{\gamma}
$$

If take $m=n=1$ and $\tau_{0}=0$ we get the Newton law.

If the yield stress $\tau_{0}$ is not equal to zero, zones of no deformation where $A$ is zero can appear in the fluid volume. To avoid the infinitely large values of the apparent viscosity $B$ instead of the equation (5) we shall use the equation

$$
B=\left(\frac{\tau_{0}}{A^{1 / m}+\epsilon}+\mu^{1 / m}\right)^{n} A^{n / m-1},
$$

where $\epsilon$ is a small parameter.

The dynamic boundary conditions at the free surface are continuity of stresses. Under the assumption that amplitudes of the oscillation are small enough compared with a wavelength these conditions can be applied at the undisturbed free surface $\Sigma_{0}$ instead of the real free surface $\Sigma$ (Fig. 2), and the $O y$ and $O x$ axes can be assumed to be normal and tangent to the free surface [3], [4]. Then the conditions at the free surface are

$$
\tau_{y y}=p, \quad \tau_{x y}=\tau_{y x}=0 .
$$

(We neglect surface tension and assume that the atmospheric pressure is zero.) The kinematic condition is

$$
\frac{\partial h}{\partial t}=v \quad \text { at } \quad \Sigma_{0},
$$


where $h(x, t)$ is the surface level above horizontal undisturbed surface $\Sigma_{0}$. (Here we omitted the convective inertia term, which is of second order under the small amplitudes assumption [3], [4].) At the solid boundary $S$ we will apply the no-slip conditions

$$
u=v=0 .
$$

As the initial conditions we can take a prescribed initial free surface position $h(x, 0)$ and a zero initial velocity field.

\section{Numerical scheme}

To solve the system (1)-(3) we will use the finite-difference scheme with splitting of the physical processes. The spatial discretization makes use of a staggered mesh (Fig. 3), which is the natural extension to non-Newtonian fluids flows of the mesh of marker-and-cell (MAC) method introduced by F.H. Harlow and J.E. Welch [2]. The mesh is staggered with the subscripts $i$ and $j$ denoting the cell centre, where $i$ counts columns in the $x$-direction, and $j$ counts rows in the $y$-direction. The unknown variables are located as displayed in Fig. 3. Such a mesh allows us to consider every rectangular cell as an element of fluid and to build a conservative numerical method.

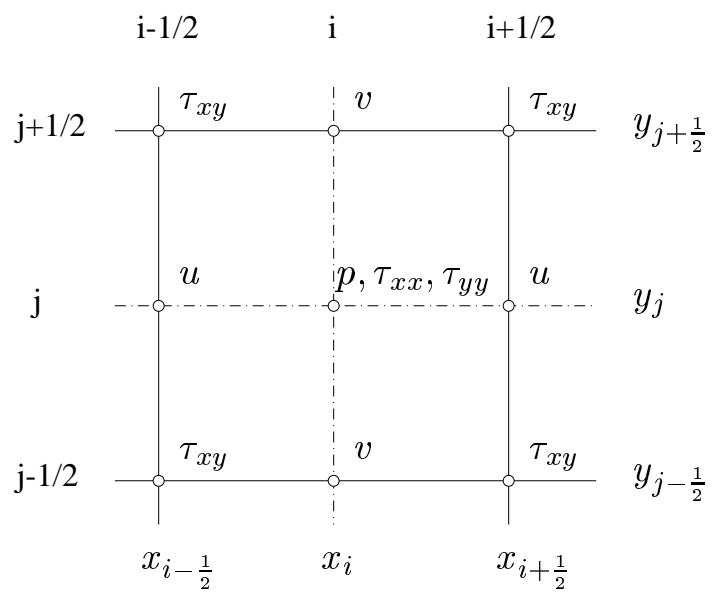

Figure 3. Mesh layout.

We will adjust an irregular rectangular mesh to the channel geometrical form (see Fig. 2). We will approximate the solid boundary $S$ by a polygonal line so that the vertices of the polygonal line coincide with the corner points of cells adjoining the boundary (Fig. 4). Thus the segments of the polygonal line will either coincide with a rectangular cell side (as for the cell $(i+1, j)$ in Fig. 4 ) or cross a cell diagonally 
forming a triangular cell (as the cells $(i-1, j+1)$ and $(i, j)$ in Fig. 4 ). Such meshes can be constructed automatically for a wide range of the channel forms. What is the more, in many cases the curve line $S$ may be defined by an sequence of its points, in which cases this approach seems to be natural. A three-dimensional generalisation of this approach is possible, although the details are not straightforward.

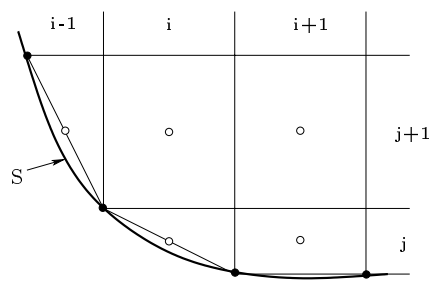

Figure 4. Approximation of a solid boundary by an irregular mesh.

Following the SMAC (simplified MAC) method by A.A. Amsden and F.H. Harlow [1], we will split each calculation cycle (a time step $\Delta t$ ) into two stages. At the first stage the momentum changes due to the fluid viscosity only. The provisional velocity field $\left(\tilde{u}^{n+1}, \tilde{v}^{n+1}\right)$ is calculated as

$$
\begin{gathered}
\rho \frac{\tilde{u}_{i+\frac{1}{2}, j}^{n+1}-u_{i+\frac{1}{2}, j}^{n}}{\Delta t}=\frac{\left(\tau_{x x}\right)_{i+1, j}^{n}-\left(\tau_{x x}\right)_{i, j}^{n}}{x_{i+1}-x_{i}}+\frac{\left(\tau_{x y}\right)_{i+\frac{1}{2}, j+\frac{1}{2}}^{n}-\left(\tau_{x y}\right)_{i+\frac{1}{2}, j-\frac{1}{2}}^{n}}{y_{j+\frac{1}{2}}-y_{j-\frac{1}{2}}}, \\
\rho \frac{\tilde{v}_{i, j+\frac{1}{2}}^{n+1}-v_{i, j+\frac{1}{2}}^{n}}{\Delta t}=\frac{\left(\tau_{x y}\right)_{i+\frac{1}{2}, j+\frac{1}{2}}^{n}-\left(\tau_{x y}\right)_{i-\frac{1}{2}, j+\frac{1}{2}}^{n}+\frac{\left(\tau_{y y}\right)_{i, j+1}^{n}-\left(\tau_{y y}\right)_{i, j}^{n}}{y_{j+\frac{1}{2}}-x_{i-\frac{1}{2}}}, y_{j}}{y_{j+1}},
\end{gathered}
$$

or in the abbreviated form

$$
\begin{gathered}
\rho \frac{\tilde{u}^{n+1}-u^{n}}{\Delta t}=\Delta_{x} \tau_{x x}^{n}+\Delta_{y} \tau_{x y}^{n}, \\
\rho \frac{\tilde{v}^{n+1}-v^{n}}{\Delta t}=\Delta_{x} \tau_{x y}^{n}+\Delta_{y} \tau_{y y}^{n} .
\end{gathered}
$$

Here the difference operators $\Delta_{x}, \Delta_{y}$ are the central differences in the $x$ - and $y$ directions respectively which approximate the partial derivatives $\frac{\partial}{\partial x}$ and $\frac{\partial}{\partial y}$. The components of the stress tensor $\tau$ are defined by the equations (4) and (6), where the deformation rates are given by the equations

$$
\begin{aligned}
\left(\dot{\gamma}_{x x}\right)_{i, j} & =\frac{u_{i+\frac{1}{2}, j}-u_{i-\frac{1}{2}, j}}{x_{i+\frac{1}{2}}-x_{i-\frac{1}{2}}} \equiv \Delta_{x} u \\
\left(\dot{\gamma}_{x y}\right)_{i+\frac{1}{2}, j+\frac{1}{2}} & =\frac{u_{i+\frac{1}{2}, j+1}-u_{i+\frac{1}{2}, j}}{2\left(y_{j+1}-y_{j}\right)}+\frac{v_{i+1, j+\frac{1}{2}}-v_{i, j+\frac{1}{2}}}{2\left(x_{i+1}-x_{i}\right)} \equiv \frac{1}{2}\left(\Delta_{y} u+\Delta_{x} v\right), \\
\left(\dot{\gamma}_{y y}\right)_{i, j} & =\frac{v_{i, j+\frac{1}{2}}-v_{i, j-\frac{1}{2}}}{y_{j+\frac{1}{2}}-y_{j-\frac{1}{2}}} \equiv \Delta_{y} v .
\end{aligned}
$$


The viscous stresses at the free surface are to be found from the difference approximation of the dynamic boundary conditions (7), namely

$$
\tau_{x y}^{n}=0, \quad \tau_{y y}^{n}=\rho\left(\Pi^{n}+g_{y} h^{n}+g_{x} x\right) \quad \text { at } \Sigma_{0},
$$

where $\Pi=p / \rho-g_{x} x-g_{y} y$ is the modified pressure, $g_{x}, g_{y}$ are components of the gravity acceleration and $x, y$ are the Cartesian coordinates of the fluid particle. At the solid boundary $S$ no-slip conditions (9) hold.

The provisional velocity field obtained after the first stage does not satisfy the finite-difference incompressibility condition, i.e.

$$
\tilde{D}^{n+1}=\Delta_{x} u^{n+\frac{1}{2}}+\Delta_{y} v^{n+\frac{1}{2}} \neq 0 .
$$

At the second stage of the splitting process the momentum changes under action of the pressure and gravity, i.e.

$$
\begin{aligned}
& \frac{u^{n+1}-\tilde{u}^{n+1}}{\Delta t}=-\Delta_{x} \Pi^{n+1}, \\
& \frac{v^{n+1}-\tilde{v}^{n+1}}{\Delta t}=-\Delta_{y} \Pi^{n+1} .
\end{aligned}
$$

The modified pressure $\Pi=p / \rho-g_{x} x-g_{y} y$ is the solution of the Poisson equation

$$
\Lambda \Pi^{n+1}=\frac{1}{\Delta t} \tilde{D}^{n+1}
$$

where the difference Laplace operator $\Lambda \equiv \Delta_{x}\left(\Delta_{x}\right)+\Delta_{y}\left(\Delta_{y}\right)$ is defined by

$$
\begin{aligned}
\Lambda \Pi & \equiv \frac{1}{x_{i+\frac{1}{2}}-x_{i-\frac{1}{2}}}\left(\frac{\Pi_{i+1, j}-\Pi_{i, j}}{x_{i+1}-x_{i}}-\frac{\Pi_{i, j}-\Pi_{i-1, j}}{x_{i}-x_{i-1}}\right) \\
& +\frac{1}{y_{j+\frac{1}{2}}-y_{j-\frac{1}{2}}}\left(\frac{\Pi_{i, j+1}-\Pi_{i, j}}{y_{j+1}-y_{j}}-\frac{\Pi_{i, j}-\Pi_{i, j-1}}{y_{j}-y_{j-1}}\right) .
\end{aligned}
$$

The equation (15) is the consequence of the equations (13), (14) and the difference equation of continuity for incompressible flow

$$
D^{n+1}=\Delta_{x} u^{n+1}+\Delta_{y} v^{n+1}=0 .
$$

The boundary conditions for the Poisson equation (15) are the dynamic boundary condition

$$
\frac{1}{\rho} \tau_{y y}^{n+1}=\Pi^{n+1}+g_{x} x+g_{y} h^{n+1} \quad \text { at } \Sigma_{0},
$$

and the Neumann condition

$$
\Delta_{n} \Pi=0
$$


(which is a consequences of the no flux condition) at the solid boundary $S$. The operator $\Delta_{n}$ approximates the partial derivative $\frac{\partial}{\partial n}$ in the direction of the outward normal to a boundary.

The position of the free surface $\Sigma$ is to be defined using the approximation of the kinematic boundary condition (8),

$$
\frac{h^{n+1}-h^{n}}{\Delta t}=v^{n+1}
$$

It is easy to see that the equivalent whole-step scheme, which can be obtained by summing the equation (10) with (13), and the equation (11) with (14), forms a second order approximation of the differential system (1), (2).

\section{Numerical examples}

The numerical scheme described in Section 3 has been applied to simulate small oscillations (the oscillations are of small amplitudes compared with wavelength) of fluids in a cross-section of a cylindrical channel. The channel radius is equal to one, and the fluids occupy half of the channel. The fluid density $\rho$ is equal to one, the gravity acceleration $g_{y}=9.8$ and the small parameter $\epsilon=10^{-5}$. All parameters here and further are dimensionless. A variety of rheological models fitting the rheological equations (4), (6) have been considered.

To initiate oscillations two approaches were used: a non-zero initial free surface $h(x, 0)$ was set, or a horizontal impulse of gravity $g_{x}$ was applied for a short time (for two-three time steps $\Delta t$ ). In both these cases the zero initial velocity field has been used. The first way is simpler, though the second seems to be more natural and allows us to find the surface form more accurately.

The simulations showed that, in the case of small oscillations, pseudoplastics and dilatant fluids do not exhibit behaviour notably different from that of Newtonian fluid. The reason for this is that in the case of small oscillations the variations of the deformation rates (and consequently of the apparent viscosity $B$ ) are too small to cause, in the absence of the yield stress $\tau_{0}$, a manifestation of the non-linearity of rheological characteristics. The most interesting results were obtained for Bingham fluids which, due to non-zero yield stress $\tau_{0}$, demonstrated behaviour qualitatively different from that of Newtonian fluids.

If the yield stress $\tau_{0}$ is non-zero, zones of no-deformation can appear in the fluid volume. For flows with a free surface such zones may be expected to appear at the free surface where stresses are minimal. Such zones of no-deformation have been observed during the simulations. The velocity profiles (Fig. 5) give the distribution of horizontal velocity $u$ through fluid depth. The velocity profiles of Bingham fluids flow (Fig. 5 (b), (c)) clearly indicate that there is a zone of no-deformation (quasisolid nucleus) at the free surface centre. This quasi-solid nucleus behaves as a solid body drifting in the fluid volume. Compare Fig. 5 (b), (c) with the velocity profile of Newtonian flow given by Fig. 5 (a). The inflection of the Newtonian flow profile 
is due to cylindrical form of the channel. The bigger the yield stress $\tau_{0}$ is the bigger this quasi-solid nucleus is.

Unlike a solid body, the size of this quasi-solid nucleus varies with the phase of oscillations: it expands while kinetic energy of the oscillations decreases, and it shrinks while kinetic energy increases. Thus for a period, the quasi-solid nucleus reaches its maximal size when the free surface deviates maximally from the horizontal undisturbed position $\Sigma_{0}$. Furthermore, while amplitudes of oscillations decrease the quasi-solid nucleus expands, until eventually, when the amplitude reaches a critical value, it seizes all fluid volume. Then the oscillation ceases and the free surface stands still in a disturbed position.

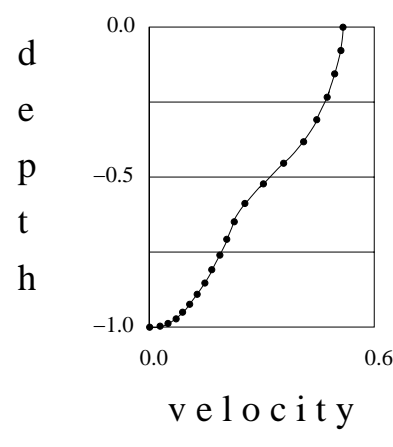

(a)

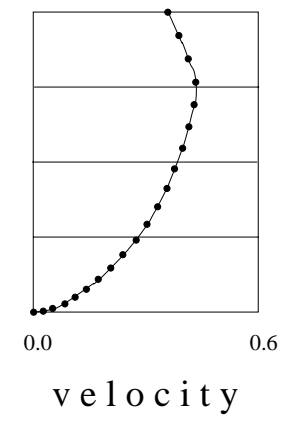

(b)

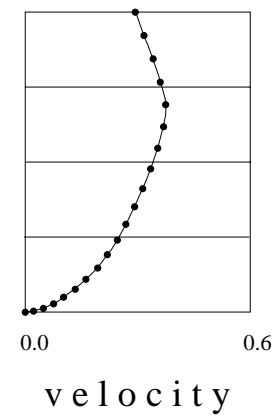

(c)

Figure 5. Velocity profiles at the plane of symmetry of a semi-cylindrical channel of radius one. Here (a) is the profile for Newtonian fluid with $\mu=0.05, \tau_{0}=0$, (b) is for Bingham fluid with $\mu=0.05, \tau_{0}=0.05$ and (c) is for Bingham fluid with $\mu=0.05, \tau_{0}=0.07$. All data for amplitude $A=0.24$.

The most notable difference between Bingham and Newtonian fluids oscillations is in the energy dissipation process. To characterise the energy dissipation at oscillations it is convenient to define the coefficient

$$
\delta=2 \ln \frac{A^{(n)}}{A^{(n+1)}},
$$

where $A^{(n)}, A^{(n+1)}$ are the amplitudes at two successive maxima of potential energy of the oscillations [3]. (That is $A^{(n)}$ and $A^{(n+1)}$ are measured with a time interval $T / 2$, where $T$ is the period of the oscillations. This explains the coefficient 2 in equation( 18).) If the coefficient $\delta$ is constant it is usually called the logarithmic decrement. In this case the full energy $E$ of the oscillator at a moment $t$ is given by the equation

$$
E(t)=E(0) \exp \left(-\delta \frac{t}{T}\right)
$$


For small oscillations of the Newtonian fluid the coefficient $\delta$ is fairly constant [3]: it does not depend on the amplitude and does not change from a period to period (see Fig. 6, curve 1). Due to the presence of the quasi-solid nucleus which expands while the amplitude decreases, the energy dissipation of the Bingham fluid oscillations is notably different. In the case of a Bingham fluid the coefficient $\delta$ grows as the amplitude decreases (Fig. 6, curves 2 and 3), till eventually the quasisolid nucleus seizes the whole fluid volume and the oscillation stops. After this the coefficient $\delta$ does not have any physical meaning.

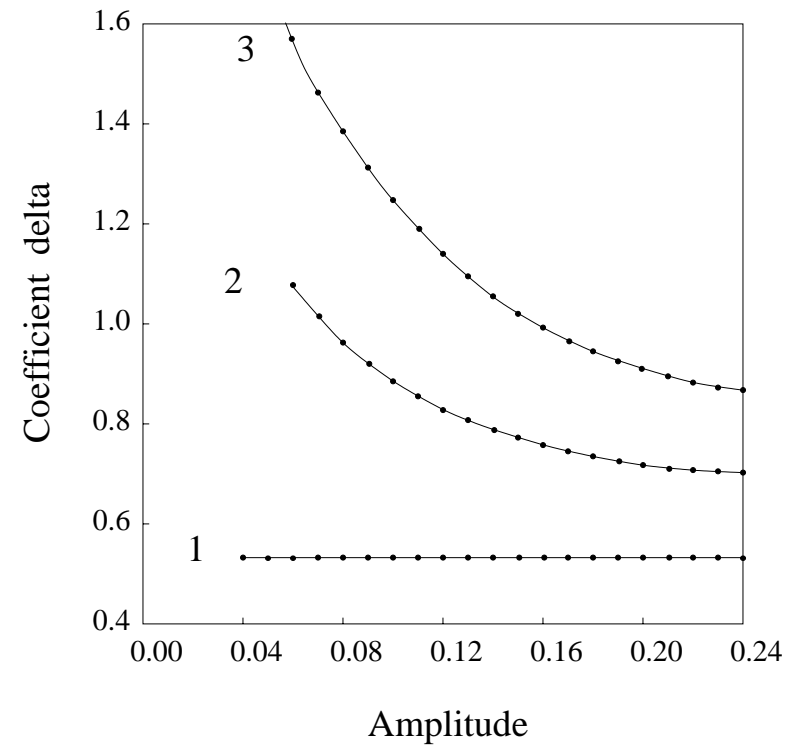

Figure 6. Coefficient $\delta$ versus amplitudes $A$ for the oscillations of Newtonian (curve 1 ) and Bingham (curves 2 and 3 ) fluids. Here curve 1 is for $\mu=0.01$ and $\tau_{0}=0$; curve 2 is for $\mu=0.01, \tau_{0}=0.004$ and curve 3 is for $\mu=0.01, \tau_{0}=0.008$.

Figures 7 and 8 demonstrate how the coefficient $\delta$ for Bingham fluids depends on the coefficient of viscosity $\mu$ (Fig. 7) and the yield stress $\tau_{0}$ (Fig. 8). It is of interest that for small oscillations, the coefficient $\delta$ depends on the yield stress $\tau_{0}$ linearly.

In the case of small oscillations the forms of a free surface do not vary noticeably with variation of the rheological characteristics. The frequencies of the oscillations do not exhibit dependence on rheological characteristics either. The frequency of Bingham fluid oscillations does not depend on the yield stress $\tau_{0}$ and coincides with the frequency for a Newtonian fluid with the same coefficient $\mu$. This fact is not surprising since the frequency depends on the free surface form (which is the same), fluid density and gravity acceleration [3], [4]. 


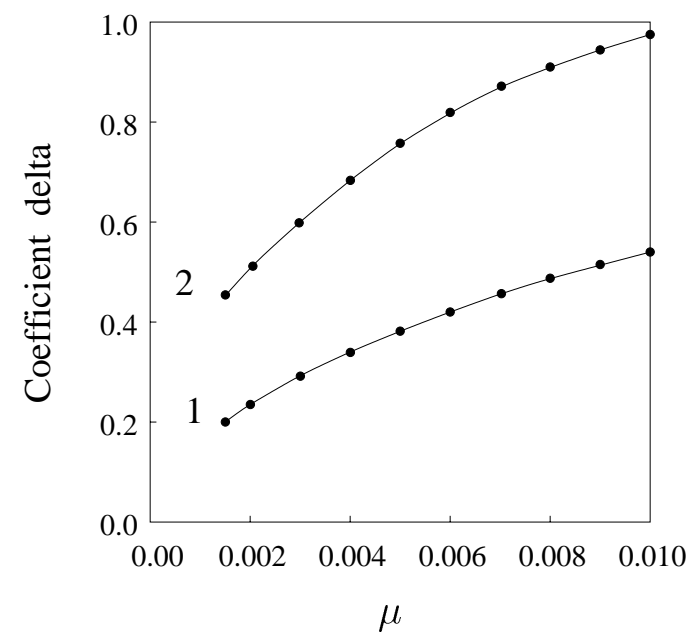

Figure \%. Coefficient $\delta$ versus the coefficient of viscosity $\mu$ for Newtonian (curve 1) and Bingham with $\tau_{0}=0.01$ (curve 2) fluids. (All data for the initial amplitude $A^{(0)}=0.24$.)

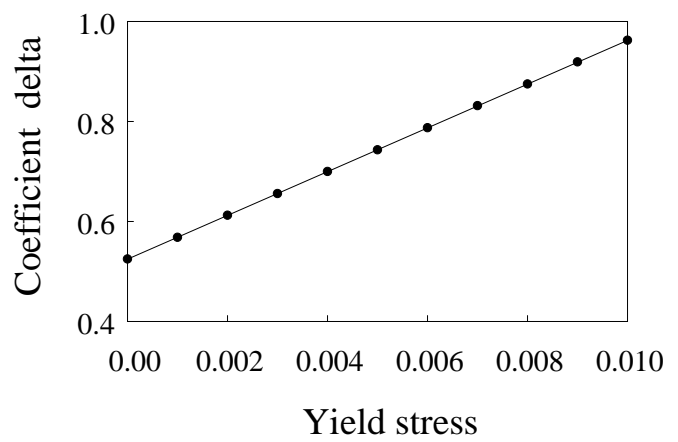

Figure 8. Coefficient $\delta$ versus the yield stress $\tau_{0}$ for Bingham fluid $(\mu=0.01$. (All data for the initial amplitude $\left.A^{(0)}=0.24\right)$. 


\section{Conclusion}

The main aim of this paper was to investigate how the non-linear rheological properties of fluids contained in a tank can affect their behaviour. The numerical simulations lead to the conclusion that, under the assumption the motion of the fluid (the amplitude of oscillation) is small enough, non-Newtonian fluids with zero yield stress do not exhibit any noticeable deviation in behaviour from Newtonian fluids. This is because for small oscillations the range of deformation rate variations throughout the fluid volume is also small and it is permissible to approximate, in the absence of the yield stress $\tau_{0}$, a non-linear rheological equation by a linear function.

If the yield stress $\tau_{0}$ is non-zero, then the fluid exhibits features qualitatively different from those of a Newtonian fluid, particularly in the energy dissipation process. Thus, the Bingham fluid oscillations stop in a disturbed free surface position, end in a finite time (whilst the Newtonian fluid oscillations continue infinitely) and exhibit a notable dependence of the energy dissipation on amplitude.

We note that all the qualitative results presented, as well as the conclusions are valid for the three-dimensional case. In this paper we considered the twodimensional flow for the sake of simplicity and because we are interested in qualitative properties.

The numerical method applied in this paper is not limited by two-dimensional flows and could be easily extended to three-dimensional flows. The method is not limited by the constitutive relationship (4), (6) used in this paper. In fact, since the rheological equations are not incorporated into the governing equations (1), (2) explicitly, any constitutive relation suitable for multi-dimensional flows (including those for time-dependent fluids) can be used.

\section{Acknowledgments}

The author thanks Dr W. Walker and Dr A. McNaughton of the University of Auckland for assistance with preparation of this paper for publication.

\section{References}

1. A.A. Amsden, F.H. Harlow. The SMAC method: a numerical technique for calculating incompressible fluid flows. Los Alamos Scientific Laboratory, Report LA-4370, 1971.

2. F.H. Harlow, J.E. Welch. Numerical calculation of time-dependent viscous incompressible flow of fluid with free surface. Phys. Fluids, 8(12): 2182-2189, 1965.

3. G.N. Mikishev, B.I. Rabinovich. Dynamics of solid body with caverns partly filled with fluid (in Russian). Mashinostroenie, Moscow, 1968.

4. N.N. Moiseev, V.V. Rumyantsev. Dynamic stability of bodies containing fluid. SpringerVerlag, New York, 1968.

5. M. Reiner. Rheology of concrete, In: Rheology: Theory and Applications, (Edited by F.R. Eirich), vol. 3, pp. 341-364, Academic Press, New York, 1960.

6. E.P. Shulman. Convective heat and mass transportation of rheologically complex fluids (in Russian). Energiya, Moscow, 1975. 
7. E.P. Shulman, B.M. Berkovskii. Boundary layer of non-Newtonian fluids (in Russian). Nauka i Tekhnika, Minsk, 1966.

8. W.L. Wilkinson. Non-Newtonian Fluids: Fluid Mechanics and Heat Transfer. Pergamon, London, 1960.

9. N.N. Yanenko. The Method of Fractional Steps. Springer-Verlag, New York, 1971. 


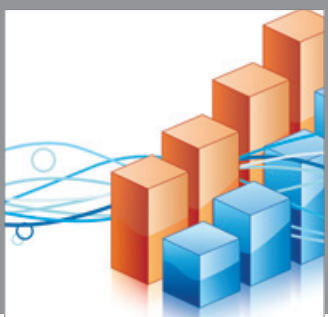

Advances in

Operations Research

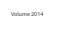

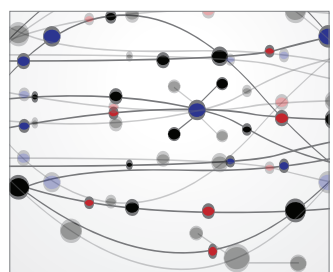

\section{The Scientific} World Journal
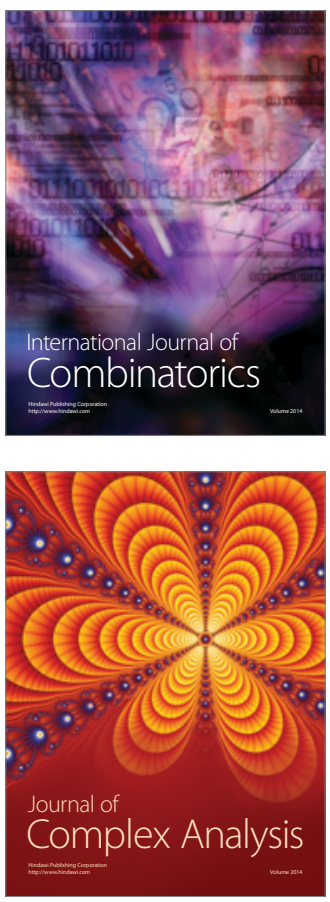

International Journal of

Mathematics and

Mathematical

Sciences
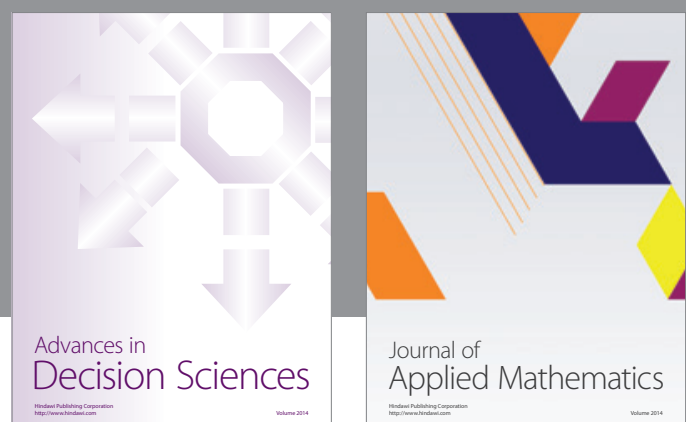

Journal of

Applied Mathematics
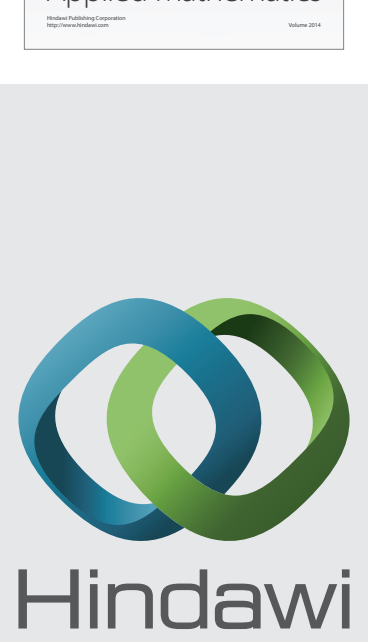

Submit your manuscripts at http://www.hindawi.com
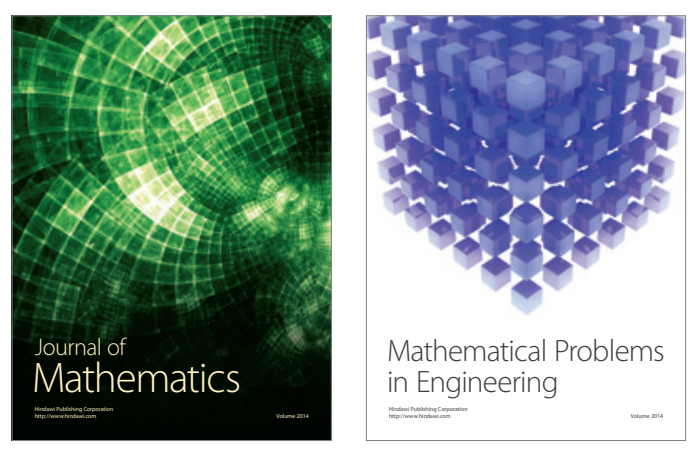

Mathematical Problems in Engineering
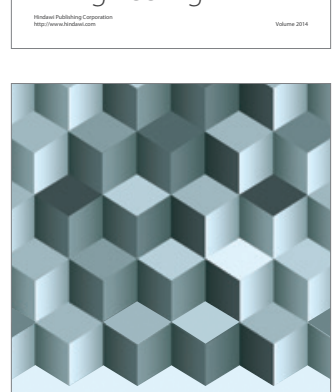

Journal of

Function Spaces
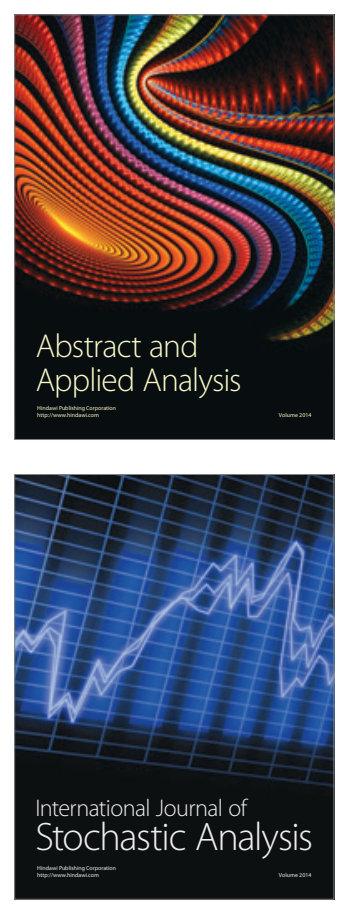

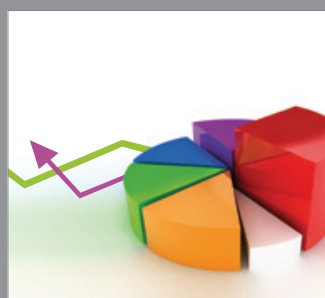

ournal of

Probability and Statistics

Promensencen
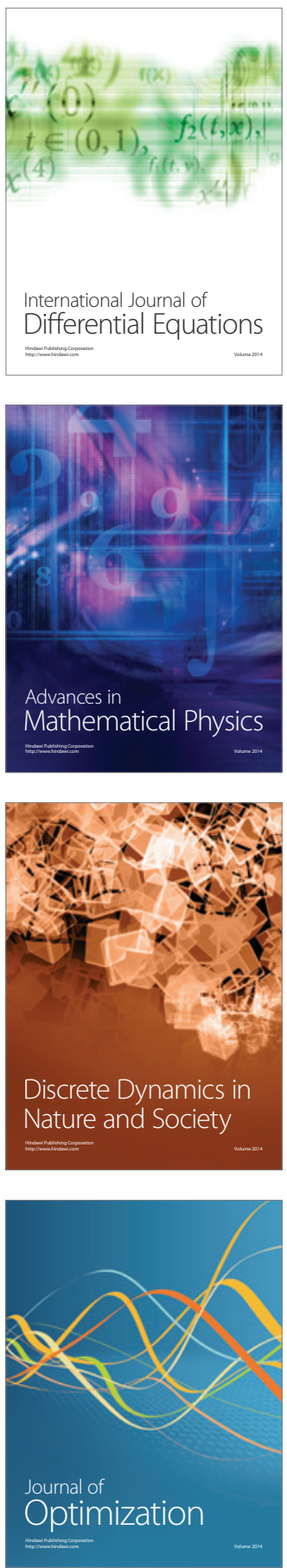